\title{
Critical behavior and magnetocaloric effect of $\mathrm{Pr}_{1-\mathrm{x}} \mathrm{Ca}_{\mathrm{x}} \mathrm{MnO}_{3}$
}

\author{
T. A. Ho, ${ }^{1}$ T. D. Thanh,,${ }^{1,2}$ Yikyung Yu, ${ }^{3}$ D. M. Tartakovsky, ${ }^{3}$ T. O. Ho, ${ }^{4}$ P. D. Thang,${ }^{5}$ \\ Anh-Tuan Le, ${ }^{6}$ The-Long Phan, ${ }^{1}$ and S. C. Yu ${ }^{1, a)}$ \\ ${ }^{1}$ Department of Physics, Chungbuk National University, Cheongju 361-763, South Korea \\ ${ }^{2}$ Institute of Materials Science, Vietnam Academy of Science and Technology, 18-Hoang Quoc Viet, Hanoi, Vietnam \\ ${ }^{3}$ Department of Mechanical and Aerospace Engineering, University of California, San Diego, \\ California 92093-0411, USA \\ ${ }^{4}$ Institute of Chemistry, Vietnam Academy of Science and Technology, 18-Hoang Quoc Viet, Hanoi, Vietnam \\ ${ }^{5}$ Faculty of Engineering Physics and Nanotechnology, VNU University of Engineering and Technology, \\ 144 Xuan Thuy, Cau Giay, Hanoi, Vietnam \\ ${ }^{6}$ Department of Nanoscience and Nanotechnology, Advanced Institute for Science and Technology, \\ Hanoi University of Science and Technology, 01 Dai Co Viet, Hai Ba Trung, Hanoi, Vietnam
}

(Presented 5 November 2014; received 18 September 2014; accepted 2 November 2014; published online 13 March 2015)

The critical behavior of $\operatorname{Pr}_{1-x} \mathrm{Ca}_{x} \mathrm{MnO}_{3}$ samples with $x=0.25,0.27$, and 0.29 has been investigated. Detailed analyses of magnetic-field dependences of magnetization at temperatures around the paramagnetic-ferromagnetic transition, $M(H, T)$, reveal that the samples undergo a second-order magnetic phase transition. The Arrott plot method predicts the values of critical parameters to be $T_{\mathrm{C}} \approx 118 \mathrm{~K}, \beta=0.351 \pm 0.003, \gamma=1.372 \pm 0.002$, and $\delta=4.90 \pm 0.02$ for $x=0.25 ; T_{\mathrm{C}} \approx 116 \mathrm{~K}$, $\beta=0.362 \pm 0.002, \quad \gamma=1.132 \pm 0.004, \quad$ and $\delta=4.09 \pm 0.03 \quad$ for $x=0.27 ; \quad$ and $T_{\mathrm{C}} \approx 110 \mathrm{~K}$, $\beta=0.521 \pm 0.002, \gamma=0.912 \pm 0.005$, and $\delta=2.71 \pm 0.02$ for $x=0.29$. The values of $\beta=0.351$ (for $x=0.25$ ) and $\beta=0.362$ (for $x=0.27$ ) are close to the value $\beta=0.365$ expected for the 3D Heisenberg model, proving an existence of short-range ferromagnetic interactions in these samples. A slight increase in Ca-doping content $(x=0.29)$ leads to the shift of the $\beta$ value $(=0.521)$ towards that of the mean-field theory (with $\beta=0.5$ ) characteristic of long-range ferromagnetic interactions. The samples also exhibit a magnetocaloric effect: around $T_{\mathrm{C}}$ of $\mathrm{Pr}_{1-x} \mathrm{Ca}_{x} \mathrm{MnO}_{3}$ compounds, magnetic-entropy change reaches the maximum values of about $5.0,4.1$, and $2.5 \mathrm{~J} \mathrm{~kg}^{-1} \mathrm{~K}^{-1}$ for $x=0.25,0.27$, and 0.29 , respectively, under an applied-field change of $50 \mathrm{kOe}$. Magnetic-field dependences of the maximum magnetic-entropy change $\left(\Delta S_{\max }\right)$ obey a power law $\left|\Delta S_{\max }(H)\right| \propto H^{n}$, where exponent values $n=0.68-0.74$ are close to those obtained from the theoretical relation $n=1+(\beta-1) /(\beta+\gamma)$. $\odot 2015$ AIP Publishing LLC. [http://dx.doi.org/10.1063/1.4914537]

The discovery of a colossal magnetoresistance and magnetocaloric (MC) effects in hole-doped perovskite-type manganites of $R_{1-x} A_{x}^{\prime}{ }_{x} \mathrm{MnO}_{3}$ (where $R$ and $A^{\prime}$ are rare-earth and alkaline-earth metals, respectively) has generated significant interest in the solid-state physics community. ${ }^{1}$ The substitution of $A^{\prime}$ for $R$ in these materials adds $\mathrm{Mn}^{4+}$ ions, pre-existing $\mathrm{Mn}^{3+}$ ions. This modifies the structural characterization and magnetic interactions of manganites. Among the studied hole-doped manganites, $\mathrm{Pr}_{1-\mathrm{x}} \mathrm{Ca}_{\mathrm{x}} \mathrm{MnO}_{3}$ compounds are most promising because their electrical and magnetic properties at temperatures below the ferromagnetic-paramagnetic (FM-PM) phase transition $\left(T_{\mathrm{C}}\right.$, the Curie temperature) associated with double-exchange (DE) interactions between $\mathrm{Mn}^{3+}$ and $\mathrm{Mn}^{4+}$ ions are intriguing and sensitive to the variation of $\mathrm{Ca}$ content $(x) .^{2-5}$ For example, in the doping range $x=0.3-0.5$, $\mathrm{Pr}_{1-\mathrm{x}} \mathrm{Ca}_{\mathrm{x}} \mathrm{MnO}_{3}$ compounds exhibit the charge-ordered antiFM behavior, while the compounds with $0<x<0.3$ are a FM insulator. These features are quite different from lanthanummanganite-based compounds, in which the FM phase is accompanied with metal-like behaviors. ${ }^{2}$

$\mathrm{Pr}_{1-\mathrm{x}} \mathrm{Ca}_{\mathrm{x}} \mathrm{MnO}_{3}$ compounds also demonstrate non-trivial magnetic interactions. For example, the field- and temperature-

${ }^{a)}$ Electronic mail: scyu@chungbuk.ac.kr. Fax: +82-43-275-6416 dependent $\mathrm{AC}$ susceptibility and magnetization for $\operatorname{Pr}_{1-\mathrm{x}}$ $\mathrm{Ca}_{\mathrm{x}} \mathrm{MnO}_{3}$ single crystals with $x=0.27$ and 0.29 , exhibit nearly Heisenberg model exponents. ${ }^{4}$ Polycrystalline $\operatorname{Pr}_{1-x}$ $\mathrm{Ca}_{\mathrm{x}} \mathrm{MnO}_{3}$ samples revealed coexistence of anti-FM/FM and spin-glass behaviors at low Ca-doping concentrations $(x=0.0-0.2){ }^{3}$ The samples with higher $\mathrm{Ca}$ concentrations (e.g., $\quad x=0.3-0.5)$ exhibit the charge-ordered state. Particularly, in the range of $x=0.2-0.3$, the samples demonstrate a non-monotonic change of $T_{\mathrm{C}}$, with the maximum value of $T_{\mathrm{C}}$ achieved for $x=0.25 .^{2}$

To further understand magnetic interactions in these compounds, we prepared polycrystalline $\mathrm{Pr}_{1-\mathrm{x}} \mathrm{Ca}_{\mathrm{x}} \mathrm{MnO}_{3}$ samples with $x=0.25,0.27$, and 0.29 , and studied their critical behavior. FM interactions of the first two samples fall into the 3D-Heisenberg universality class, while those of the third $(x=0.29)$ fall into the universality class of the meanfield theory. Additionally, the MC effect of these compounds is also taken into account.

Three polycrystalline samples $\mathrm{Pr}_{1-\mathrm{x}} \mathrm{Ca}_{\mathrm{x}} \mathrm{MnO}_{3}$ with $x=0.25,0.27$, and 0.29 were prepared from high-purity powders of $\operatorname{Pr}_{6} \mathrm{O}_{11}, \mathrm{CaCO}_{3}$, and $\mathrm{MnO}(3 \mathrm{~N})$ using conventional solid-state reaction. The powders combined with stoichiometric quantities were mixed, ground carefully, and then pre-annealed at $1100^{\circ} \mathrm{C}$ for $24 \mathrm{~h}$. After pre-annealing, the 
mixtures were re-ground, pressed into pellets, and sintered in air at $1300{ }^{\circ} \mathrm{C}$ for $48 \mathrm{~h}$. An X-ray diffractometer (Brucker AXS, D8 Discover) was used to check the crystal structure of the final products. Room-temperature X-ray diffraction patterns revealed the samples exhibiting a single phase in an orthorhombic structure (space group: Pbnm). Magnetization measurements versus temperature and magnetic field were performed on a superconducting quantum interference device (SQUID) working at temperatures ranging from 5 to $350 \mathrm{~K}$.

To study the influence of $\mathrm{Ca}$ doping on the magnetic properties of $\mathrm{Pr}_{1-\mathrm{x}} \mathrm{Ca}_{\mathrm{x}} \mathrm{MnO}_{3}$, we measured temperature dependences of zero-field-cooled (ZFC) and field-cooled (FC) magnetizations, $M_{\mathrm{ZFC} / \mathrm{FC}}(T)$, for an applied field $H=100$ Oe. The results shown in Fig. 1 indicate that with increasing temperature, $M_{\mathrm{ZFC}}$ first increases and then reaches its maximum value (where magnetic energy of anisotropic fields generated from FM clusters/domains is equal to that of the external field $H=100 \mathrm{Oe}$ ). Increasing temperature above $110 \mathrm{~K}$ (for $x=0.25$ and 0.27 ) and $90 \mathrm{~K}$ (for $x=0.29$ ) rapidly reduces $M_{\mathrm{ZFC}}$ values due to the FM-PM phase transition, in which the $\mathrm{Mn}^{3+}-\mathrm{Mn}^{4+} \mathrm{FM}$ coupling is weakened by thermal activation energy. The $T_{\mathrm{C}}$ values determined from the minima of $\mathrm{d} M(T) / \mathrm{d} T$ versus $T$ curves (see the insets of Fig. 1) are about $124 \mathrm{~K}, 118 \mathrm{~K}$, and $110 \mathrm{~K}$ for $x=0.25,0.27$, and 0.29 , respectively. It appears that $T_{\mathrm{C}}$ slightly shifts towards lower temperatures when the $\mathrm{Ca}$ concentration in $\operatorname{Pr}_{1-x} \mathrm{Ca}_{\mathrm{x}} \mathrm{MnO}_{3}$ increases. This is because the substitution of $\mathrm{Ca}^{2+}$ for $\mathrm{Pr}^{3+}$ introduces more $\mathrm{Mn}^{4+}$ ions, which enhance $\mathrm{Mn}^{4+}-\mathrm{Mn}^{4+}$ anti-FM interactions besides pre-existing $\mathrm{Mn}^{3+}-\mathrm{Mn}^{3+}$ antiFM ones. The reinforcement of anti-FM interactions weakens $\mathrm{Mn}^{3+}-\mathrm{Mn}^{4+} \mathrm{FM}$ interactions, and thus decreases the phase-transition temperature $T_{\mathrm{C}}{ }^{2-4}$ Specifically, the $M_{\mathrm{ZFC}}$ and $M_{\mathrm{FC}}$ branches bifurcated at $T=118,112$, and $100 \mathrm{~K}$ for $x=0.25,0.27$, and 0.29 , respectively. At $T=5 \mathrm{~K}$, this deviation is about $10 \mathrm{emu} / \mathrm{g}$, which gradually decreases at higher temperatures. This might be due to the existence of an anisotropic field generated by FM clusters. Magnetic moments of the $\mathrm{Mn}$ ions could be frozen in the directions favored energetically by their local anisotropy field or by an external field. It has also been suggested that this feature is related to the coexistence of FM and anti-FM phases and magnetic frustration existing in the samples. ${ }^{6}$

The present study focuses on the critical behavior of the $\operatorname{Pr}_{1-}$ ${ }_{\mathrm{x}} \mathrm{Ca}_{\mathrm{x}} \mathrm{MnO}_{3}$ samples. Figure 2 shows the $M(H)$ curves, and Arrott plots $^{7}\left(M^{2}\right.$ versus $\left.H / M\right)$ at temperatures around the $T_{\mathrm{C}}$ values.

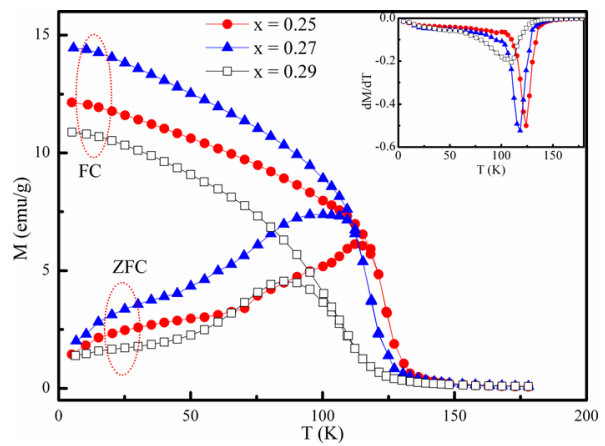

FIG. 1. $M_{\mathrm{ZFC} / \mathrm{FC}}(T)$ curves for $\operatorname{Pr}_{1-\mathrm{x}} \mathrm{Ca}_{\mathrm{x}} \mathrm{MnO}_{3}$ with $x=0.25,0.27$, and 0.29 in the field $H=100 \mathrm{Oe}$.
The $M(H)$ curves are typical for ferromagnets. Magnetization increases quickly at low-magnetic fields, and then increases slowly at fields $H>10 \mathrm{kOe}$. However, no saturation magnetization is achieved for all the $M(H)$ curves, even though high fields (up to $50 \mathrm{kOe}$ ) are used. This might be due to the existence of magnetic inhomogeneity or short-range FM order. Such the behaviors were also observed for $\mathrm{Pr}_{0.7} \mathrm{Ca}_{0.3} \mathrm{MnO}_{3}{ }^{8}$

The Arrott plots in Figs. 2(b), 2(d), 2(f) exhibit nonlinear $M^{2}-H / M$ parts in the low-field region, which are driven in two opposite directions due to the FM-PM phase separation. The inverse Arrott plots $\left(H / M\right.$ versus $M^{2}$ curves, not show here) have positive slopes. This assures that the samples undergo a second-order magnetic phase transition (SOPT), according to Banerjee's criteria. ${ }^{7}$ For a ferromagnet with the SOPT, temperature dependences of the spontaneous magnetization $\left(M_{\mathrm{s}}\right)$, inverse initial susceptibility $\left(\chi_{0}^{-1}\right)$, and critical isotherm at $T=T_{\mathrm{C}}(M)$ obey asymptotic relations ${ }^{9}$

$$
\begin{aligned}
M_{\mathrm{s}}(T) & =M_{0}(-\varepsilon)^{\beta}, \quad \varepsilon<0, \\
\chi_{0}^{-1}(T) & =\left(h_{0} / M_{0}\right) \varepsilon^{\gamma}, \quad \varepsilon>0, \\
M & =D H^{1 / \delta}, \quad \varepsilon=0,
\end{aligned}
$$

where $\varepsilon=\left(T-T_{C}\right) / T_{C}$ is the reduced temperature, $M_{0}, h_{0} / M_{0}$, and $D$ are the critical amplitudes. $\beta, \gamma$, and $\delta$ are the critical exponents associated with $M_{\mathrm{s}}, \chi_{0}{ }^{-1}$, and $M$, respectively. The exponents $\beta$ and $\gamma$ quantify the growth of ordered moments at $T<T_{\mathrm{C}}$ and the divergence of the magnetic susceptibility at $T>T_{\mathrm{C}}$, respectively. ${ }^{10} \mathrm{We}$ used Eqs. (1) and (2), and the static-scaling equation of state $(H / M)^{1 / \gamma}=a \varepsilon+b M^{1 / \beta}$ (where $a$ and $b$ are temperature-dependent parameters) to determine values of $\beta$ and $\gamma$ (this approach is known as the modified Arrott plots). ${ }^{9}$ According to this method, correct values of $\beta$ and $\gamma$ results in the $M^{1 / \beta}$ versus $(H / M)^{1 / \gamma}$ curves that are straight and parallel to each other in the vicinity of $T_{\mathrm{C}}$. The $M_{\mathrm{s}}(T)$ and $\chi_{0}{ }^{-1}(T)$ data are determined from the intersections of

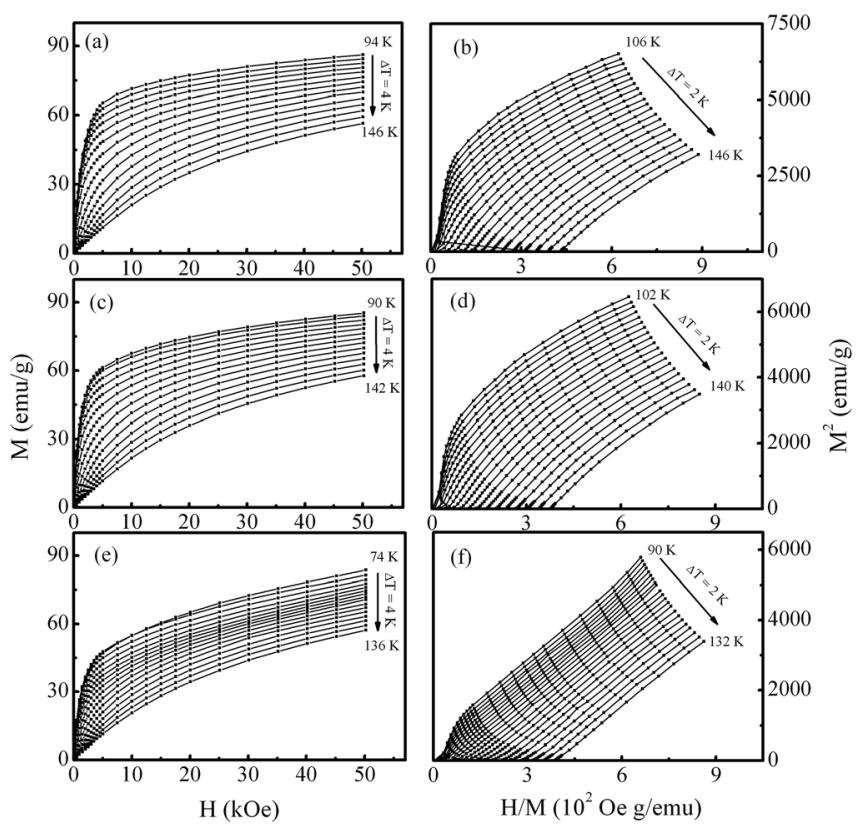

FIG. 2. $M(H)$ curves and Arrott plots $\left(M^{2}\right.$ versus $\left.H / M\right)$ for $\operatorname{Pr}_{1-\mathrm{x}} \mathrm{Ca}_{\mathrm{x}} \mathrm{MnO}_{3}$ with (a) and (b) $x=0.25$; (c) and (d) $x=0.27$; and (e) and (f) $x=0.29$. 
linear extrapolations at high-magnetic fields with the $M^{1 / \beta}$ and $(H / M)^{1 / \gamma}$ axes, respectively. Thus determined $M_{\mathrm{s}}(T)$ and $\chi_{0}{ }^{-1}(T)$ data are then fitted to Eqs. (1) and (2), respectively, to improve the estimates of $\beta, \gamma$, and $T_{C}$. New values of these critical parameters are subsequently re-used in next modified Arrott plots. This process is repeated until the $\beta, \gamma$, and $T_{C}$ values converge to stable ones. This procedure yields $T_{\mathrm{C}} \approx 118 \mathrm{~K}, \beta=0.351$ \pm 0.003 , and $\gamma=1.372 \pm 0.002$ for $x=0.25 ; T_{\mathrm{C}} \approx 116 \mathrm{~K}$, $\beta=0.362 \pm 0.002$, and $\gamma=1.132 \pm 0.004$ for $x=0.27$; and $T_{\mathrm{C}} \approx 110 \mathrm{~K}, \quad \beta=0.521 \pm 0.002$, and $\gamma=0.912 \pm 0.005$ for $x=0.29$. The results of the best fitting are shown in Fig. 3 .

The critical exponent $\delta$ is determined by fitting the critical isotherm $M$ at the critical temperature $T_{\mathrm{C}}$ to Eq. (3). Its values are $4.90 \pm 0.02,4.09 \pm 0.03$, and $2.71 \pm 0.02$ for the samples with $x=0.25,0.27$, and 0.29 , respectively. These values are close to those obtained by using the Widom scaling relation $\delta=1+\gamma / \beta(4.91,4.12$, and 2.76 for $x=0.25$, 0.27 , and 0.29 , respectively).

The scaling law ${ }^{11}$ stipulates that $M(H, T)$ data in the vicinity of $T_{\mathrm{C}}$ obey the scaling expression $M(H, \varepsilon)=|\varepsilon|^{\beta} f_{ \pm}$ $\left(H /|\varepsilon|^{\beta+\gamma}\right)$, where $f_{+}$and $f_{-}$are analytic functions associated with temperatures above and below $T_{\mathrm{C}}$, respectively. This equation implies that with correct $\beta$ and $\gamma$ values, the $M /|\varepsilon|^{\beta}$ versus $H /|\varepsilon|^{(\beta+\gamma)}$ data fall into two universal branches, one for temperatures $T>T_{\mathrm{C}}$ and the other for $T<T_{\mathrm{C}}$. Figure 4 shows that the $M(H, T)$ data for all samples, plotted in linear and logarithmic scales, fall into two universal curves for $T<T_{C}$ and $T>T_{C}$. This demonstrates that the obtained critical values are reliable and support the scaling hypothesis. It should be noticed that both the modified Arrott plots method and the scaling law work well for high-magnetic fields $(H>10 \mathrm{kOe})$. Their performance deteriorates in low-field regions because of the rearrangement of magnetic domains and uncertainty in the calculation of demagnetization factor. ${ }^{12}$

The critical values determined from our $\operatorname{Pr}_{1-x} \mathrm{Ca}_{x} \mathrm{MnO}_{3}$ samples, theoretical models, ${ }^{13,14}$ and similar materials are listed

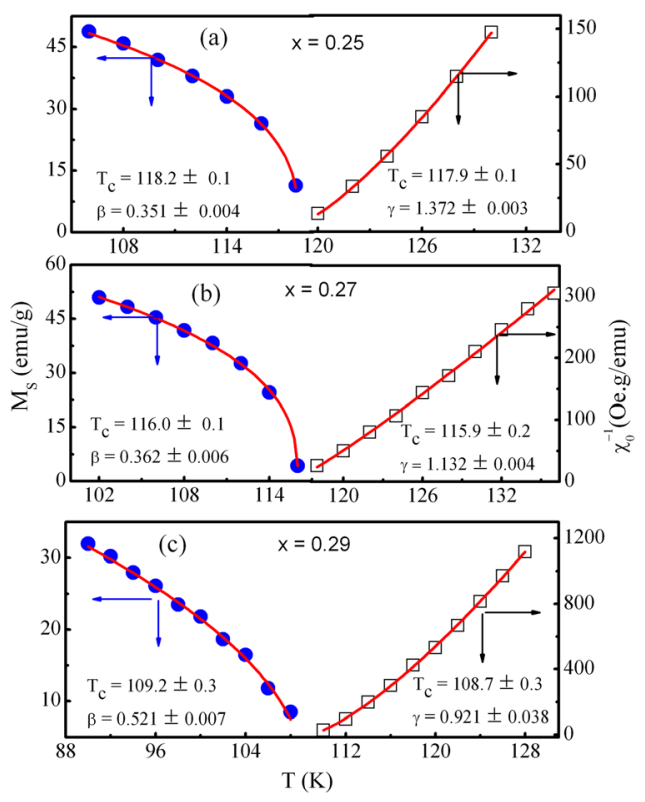

FIG. 3. $M_{\mathrm{S}}(T)$ and $\chi_{0}{ }^{-1}(T)$ data fitted to Eqs. (1) and (2), respectively, for $\operatorname{tPr}_{1-\mathrm{x}} \mathrm{Ca}_{\mathrm{x}} \mathrm{MnO}_{3}$ with (a) $x=0.25$, (b) $x=0.27$, and (c) $x=0.29$.
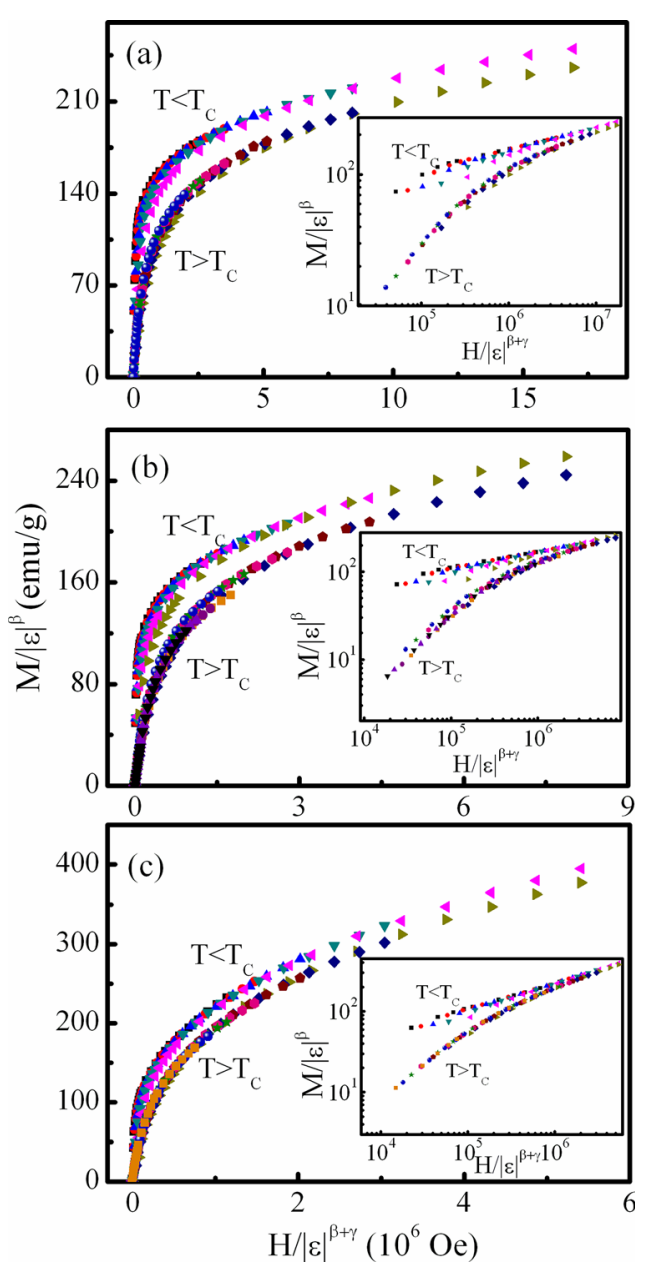

FIG. 4. Scaling performances of $M /|\varepsilon|^{\beta}$ versus $H /|\varepsilon|^{\beta+\gamma}$ in the linear scale at temperatures $T<T_{\mathrm{C}}$ and $T>T_{\mathrm{C}}$ for $\operatorname{Pr}_{1-\mathrm{x}} \mathrm{Ca}_{\mathrm{x}} \mathrm{MnO}_{3}$ with (a) $x=0.25$, (b) $x=0.27$, and (c) $x=0.29$. The insets plot the same data in the logarithmic scale.

in Table I. The critical exponent values for the $x=0.25$ and 0.27 samples are close to those expected for the 3D-Heisenberg model. Meanwhile, those for $x=0.29$ are close to the meanfield theory. Similar behavior was found in some compounds studied previously, such as $\operatorname{Pr}_{1-x} \mathrm{Ca}_{x} \mathrm{MnO}_{3}$ compounds with $x=0.27$ and 0.29 , and $\mathrm{Pr}_{0.77} \mathrm{~Pb}_{0.23} \mathrm{MnO}_{3}{ }^{4,15}$ This demonstrates the presence of a short-range FM order in the $x=0.25$ and 0.27 samples, and a long-range FM order in the $x=0.29$ sample.

In addition to the critical behavior of the $\operatorname{Pr}_{1-x} \mathrm{Ca}_{x} \mathrm{MnO}_{3}$ samples, we also analyzed their $\mathrm{MC}$ effect by studying magnetic-entropy change $\left(\Delta S_{\mathrm{m}}\right)$ as a function of temperature and magnetic field. With ferromagnets undergoing the SOPT, $\Delta S_{\mathrm{m}}$ can be calculated from the Maxwell relation as ${ }^{16}$

$$
\Delta S_{m}(T, H)=\int_{0}^{H}\left(\frac{\partial M}{\partial T}\right)_{H} d H,
$$

where $H$ is the applied field. According to Eq. (4), the magnitude of $\Delta S_{\mathrm{m}}$ depends not only on the magnitude of the magnetization $M$, but also on $(\partial M / \partial T)_{H}$. Since $(\partial M / \partial T)_{H}$ is related to magnetic ordering transition, a sharp change in the $M(T)$ curve at the FM-PM phase transition leads to a large $\Delta S_{\mathrm{m}}$ value. ${ }^{17}$ Figure 5(a) shows the $\left|\Delta S_{\mathrm{m}}(T)\right|$ curves for the $\mathrm{Pr}_{1-\mathrm{x}} \mathrm{Ca}_{\mathrm{x}} \mathrm{MnO}_{3}$ samples in an applied-field interval $\Delta H=50 \mathrm{kOe}$. A maximum 
TABLE I. Comparison of critical exponents of our $\mathrm{Pr}_{1-x} \mathrm{Ca}_{\mathrm{x}} \mathrm{MnO}_{3}$ compounds with different theoretical models, as well as other materials $(\mathrm{MAP}=$ modified Arrott plot and $\mathrm{KF}=$ Kouvel-Fisher method).

\begin{tabular}{|c|c|c|c|c|c|c|}
\hline Material & Technique & $T_{\mathrm{C}}(\mathrm{K})$ & $\beta$ & $\gamma$ & $\delta$ & References \\
\hline Mean field & Theory & $\ldots$ & 0.5 & 1.0 & 3.0 & 13 \\
\hline 3D Heisenberg & Theory & $\ldots$ & 0.365 & 1.386 & 4.8 & 13 \\
\hline 3D Ising & Theory & $\ldots$ & 0.325 & 1.241 & 4.82 & 13 \\
\hline Tricritical mean field & Theory & $\ldots$ & 0.25 & 1.0 & 5.0 & 14 \\
\hline $\operatorname{Pr}_{0.75} \mathrm{Ca}_{0.25} \mathrm{MnO}_{3}$ & MAP & 118 & $0.351 \pm 0.003$ & $1.372 \pm 0.002$ & $4.90 \pm 0.02$ & This work \\
\hline $\mathrm{Pr}_{0.73} \mathrm{Ca}_{0.27} \mathrm{MnO}_{3}$ & MAP & 116 & $0.362 \pm 0.002$ & $1.132 \pm 0.004$ & $4.09 \pm 0.03$ & This work \\
\hline $\mathrm{Pr}_{0.71} \mathrm{Ca}_{0.29} \mathrm{MnO}_{3}$ & MAP & 110 & $0.521 \pm 0.002$ & $0.912 \pm 0.005$ & $2.71 \pm 0.02$ & This work \\
\hline $\mathrm{Pr}_{0.73} \mathrm{Ca}_{0.27} \mathrm{MnO}_{3}$ & MAP & 127 & 0.36 & 1.36 & 4.81 & 4 \\
\hline $\mathrm{Pr}_{0.71} \mathrm{Ca}_{0.29} \mathrm{MnO}_{3}$ & MAP & 114 & 0.37 & 1.38 & 4.62 & 4 \\
\hline $\mathrm{Pr}_{0.77} \mathrm{~Pb}_{0.23} \mathrm{MnO}_{3}$ & $\mathrm{KF}$ & 167 & 0.344 & 1.352 & 4.69 & 15 \\
\hline $\mathrm{La}_{0.8} \mathrm{~Pb}_{0.2} \mathrm{MnO}_{3}$ & MAP & 291 & 0.499 & 1.241 & 3.49 & 19 \\
\hline
\end{tabular}
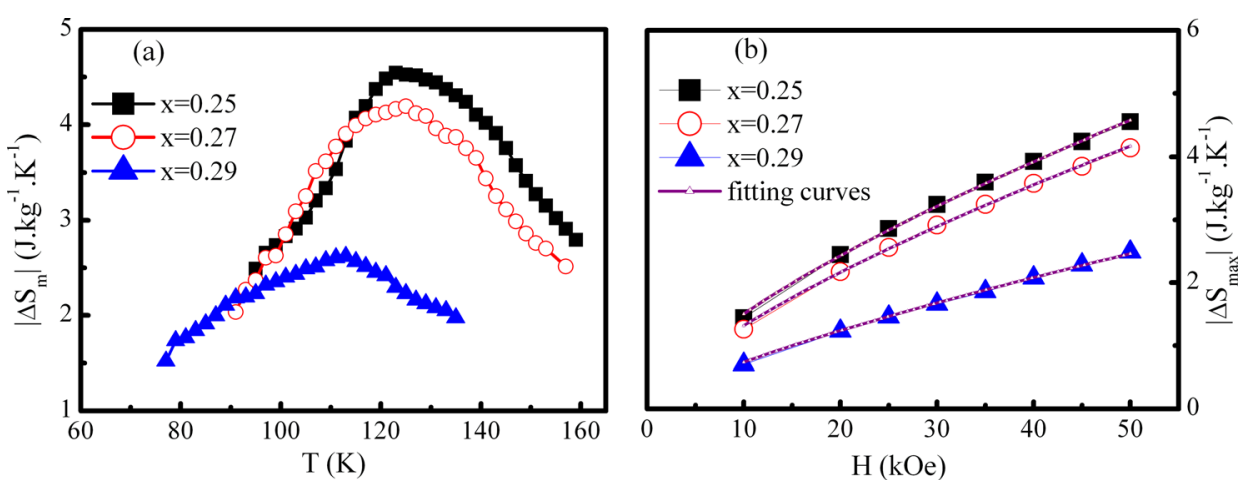

FIG. 5. $\left|\Delta S_{\max }(T)\right|$ curves for $\Delta H=50$ kOe and field dependences of $\left|\Delta S_{\max }\right|$ at $T_{\mathrm{C}}$ are fitted to a power law of $\left|\Delta S_{\max }\right| \propto H^{\mathrm{n}}$ for $\operatorname{Pr}_{1-x} \mathrm{Ca}_{x} \mathrm{MnO}_{3}$. magnetic-entropy change $\left(\left|\Delta S_{\max }\right|\right)$ occurs around $T_{\mathrm{C}}$ of the $\operatorname{Pr}_{1-x} \mathrm{Ca}_{x} \mathrm{MnO}_{3}$ samples. The magnitudes of $\left|\Delta S_{\max }\right|$ are about 5.0, 4.1, and $2.5 \mathrm{~J} \mathrm{~kg}^{-1} \mathrm{~K}^{-1}$ for $x=0.25,0.27$, and 0.29 , respectively. Similar to the $T_{\mathrm{C}}$ variation, an increase in Cadoping concentration reduces the magnitude of $\left|\Delta S_{\mathrm{m}}\right|$.

Figure 5(b) shows that the dependence of $\left|\Delta S_{\max }\right|$ on magnetic field $H$ can be described by a power law $\left|\Delta S_{\max }\right|$ $\propto H^{n}$, where $n$ is a magnetic-ordering parameter. ${ }^{16}$ The $n$ values are about $0.68,0.71$, and 0.74 for the $x=0.25,0.27$, and 0.29 samples, respectively. These values are close to those $(n=0.61,0.60$, and 0.66 for $x=0.25,0.27$, and 0.29 , respectively) calculated from the relation $n=1+(\beta-1) /(\beta+\gamma)$ proposed by Franco et al. ${ }^{16}$ Accounting for the full-width-at-half maximum of the $\left|\Delta S_{m}(T)\right|$ curve (denoted as $\delta T_{\mathrm{FWHM}}$ ) allows one to calculate the relative cooling power $(R C P)$ from the relation $R C P=\left|\Delta S_{\max }\right| \times \delta T_{\text {FWHM. }}{ }^{17}$ For $\Delta H=50 \quad \mathrm{kOe}$,

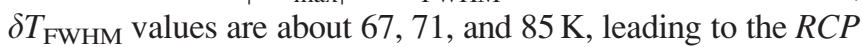
values of about 335,290 , and $215 \mathrm{~J} \mathrm{~kg}^{-1}$ for $x=0.25,0.27$, and 0.29 , respectively. These $R C P$ values are comparable to those determined from some perovskite manganites. ${ }^{17,18}$

The critical behavior and $\mathrm{MC}$ effect of $\operatorname{Pr}_{1-x} \mathrm{Ca}_{x} \mathrm{MnO}_{3}$ $(x=0.25,0.27$, and 0.29$)$ are investigated. With the values of critical parameters obtained by using the modified Arrott plot, the $M(H, T)$ point data fall into two universal branches characterized by temperatures below and above $T_{\mathrm{C}}$. The critical-exponent values of the $\operatorname{Pr}_{1-\mathrm{x}} \mathrm{Ca}_{\mathrm{x}} \mathrm{MnO}_{3}$ samples with $x=0.25$ and 0.27 are consistent with predictions of a nearest-neighbor 3D-Heisenberg model for short-range FM interactions. The critical-exponent values of the $x=0.29$ sample are close to those predicted by the mean-field theory for long-range FM interactions. Around $T_{\mathrm{C}}$, field dependences of $\left|\Delta S_{\max }\right|$ can be described by a power law $\left|\Delta S_{\max }\right|$ $\propto H^{n}$. The $n$ values $(n=0.68-0.74)$ obtained from the power-law fitting are close to those $(n=0.61,0.60$, and $0.66)$ calculated from the theoretical relation $n=1+(\beta-1) /$ $(\beta+\gamma)$. Under an applied-field change of $\Delta H=50 \mathrm{kOe}$, $\left|\Delta S_{\max }\right|$ is approximately $5.0,4.1$, and $2.5 \mathrm{~J} \mathrm{~kg}^{-1} \mathrm{~K}^{-1}$, corresponding to $R C P$ values of about 335,290 , and $215 \mathrm{~J}_{\mathrm{kg}} \mathrm{kg}^{-1}$ for the $x=0.25,0.27$, and 0.29 samples, respectively.

This research was supported by the Converging Research Center Program through the Ministry of Science, ICT and Future Planning, South Korea (2014048835).

${ }^{1}$ R. von Helmolt et al., Phys. Rev. Lett. 71, 2331 (1993).

${ }^{2}$ L. M. Fisher et al., Phys. Rev. B 68, 174403 (2003).

${ }^{3}$ T. Elovaara et al., J. Phys.: Condens. Matter 24, 216002 (2012).

${ }^{4}$ J. Wanjun et al., Phys. Rev. B 78, 144409 (2008).

${ }^{5}$ E. Pollert et al., J. Phys. Chem. Solids 43(12), 1137 (1982).

${ }^{6} \mathrm{~J}$. Wu and C. Leighton, Phys. Rev. B 67, 174408 (2003).

${ }^{7}$ B. K. Banerjee, Phys. Lett. 12, 16 (1964).

${ }^{8}$ G. E. Fedorov et al., Phys. Status Solidi B 236(2), 437 (2003).

${ }^{9}$ A. Arrott and J. E. Noakes, Phys. Rev. Lett. 19, 786 (1967).

${ }^{10}$ Z. G. Zheng et al., J. Appl. Phys. 111, 07A922 (2012).

${ }^{11}$ U. K. Rößler et al., Phys. Rev. B 70, 104417 (2004).

${ }^{12}$ T. L. Phan et al., Solid State Commun. 184, 40 (2014).

${ }^{13}$ S. N. Kaul, J. Magn. Magn. Mater. 53(1-2), 5 (1985).

${ }^{14}$ K. Huang, Statistical Mechanics, 2nd ed. (Wiley, New York, 1987).

${ }^{15}$ B. Padmanabhan et al., Phys. Rev. B 75(2), 024419 (2007).

${ }^{16}$ V. Franco et al., J. Appl. Phys. 100, 083903 (2006).

${ }^{17}$ N. S. Bingham et al., J. Appl. Phys. 106(2), 023909 (2009).

${ }^{18}$ M. H. Phan and S. C. Yu, J. Magn. Magn. Mater. 308(2), 325 (2007).

${ }^{19} \mathrm{M}$. Borowski, Perovskites: structure, properties and uses (Nova Science, 2010). 